\title{
Sperm parameters and anti-Müllerian hormone remain stable with Helicobacter pylori infection: a cross-sectional study
}

Chun Feng ${ }^{1+}{ }^{\mathbb{C}}$, Ping-Ping $\mathrm{Lv}^{2 \dagger}$, Chang-Chang Huang ${ }^{1}$, Song-Qing Yang ${ }^{1}$, Qiu-Ping Yao ${ }^{1}$, Jin-Ming Shen ${ }^{3 *}$ and Min $\operatorname{Jin}^{1 *}$ (1)

\begin{abstract}
Background and aims: It has been reported that Helicobacter pylori (HP) infection was more prevalent in infertile populations. HP infection could lead to decreased sperm parameters, and treating the HP infection could improve the quality of sperm. However, studies investigating the relationship between infertility and HP infection are still limited, and more evidence is required. Therefore, we performed the present study to investigate the impact of HP infection on sperm quality in males and on ovarian reserve in females.

Methods: A total of 16,522 patients who visited the Second Hospital of Zhejiang University from January 2016 to June 2019 due to abdominal discomfort and underwent a ${ }^{13 / 14} \mathrm{C}$-urea breath HP test were included in this retrospective cross-sectional study. Among them, 565 had performed sperm analysis or ovarian reserve tests in the past three months and were involved for further analyses. Sperm parameters were examined with a computer-assisted sperm analysis system, and serum anti-Müllerian hormone $(\mathrm{AMH})$ and sex hormones were tested with an electrochemiluminescence method.

Results: Among 363 patients who underwent the sperm test, 136 (37.47\%) had HP infection. Among 202 patients who underwent the AMH test, 55 (27.23\%) had HP infection. There was no difference in sperm concentration and motility between the HP+ and HP- groups $(P>0.05)$. Further subgroup analyses stratified into 5 -year age groups confirmed that there was no significant difference in sperm parameters $(P>0.05)$. When pooled with previously published data, no significant difference in sperm concentration or motility was found $(P>0.05)$. Meanwhile, this study found that the serum AMH level was similar between the HP+ and HP- groups $(P>0.05)$. Further subgroup analyses confirmed that there was no significant difference in serum AMH level $(P>0.05)$.
\end{abstract}

Conclusions: There were no differences in sperm parameters and AMH levels based on history of HP infection among Chinese patients.

Keywords: Helicobacter pylori (HP), Anti-müllerian hormone (AMH), Sperm parameters, Progressive motility

*Correspondence: shenjinmg@gmail.com; min_jin@zju.edu.cn

${ }^{\dagger}$ Chun Feng and Ping-Ping Lv contributed equally to this work 1 Department of Reproductive Medicine, The Second Affiliated Hospital of Zhejiang University School of Medicine, 88 Jiefang Road, Hangzhou 310009, Zhejiang, China

${ }^{3}$ Department of Orthopedics, The First Affiliated Hospital of Zhejiang

Chinese Medicine University, 54 Youdian Road, Hangzhou 310006,

Zhejiang, China

Full list of author information is available at the end of the article

\section{Background}

Currently, it is widely accepted that Helicobacter pylori (HP) may be related to a series of extragastric diseases, including cardiovascular, neurologic, respiratory, hematologic, metabolic, dermatologic, obstetric, autoimmune, and kidney diseases [1,2]. Among them, the impact of HP on fertility has attracted much attention. As early as 
20 years ago, a study in Italy suggested that the prevalence of HP infection was significantly higher in an infertile population than in controls, and antibodies against HP could be found in follicular fluids, semen, and vaginal secretions [3]. Ten years ago, a study in Japan found that the seropositive rate of HP in an infertile population with unknown etiology was higher than that in a population with known infertility factors, indicating that HP infection could be the cause of infertility [4]. In a cytotoxin-associated gene A (CagA)-positive population, the incidence of early pregnancy loss (EPL) after assisted reproductive technology increased significantly [5]. Recently, studies about infertility have focused on the impact of HP infection on sperm quality.

The first study from Italy reported a lower sperm quality in HP-infected patients with idiopathic infertility than in $\mathrm{HP}$-uninfected patients. In CagA-positive patients, both sperm motility and fertility index are reduced [6, 7]. It has been suggested that anti-CagA antibodies might block spermatozoa acrosomes and disturb fertilization [8]. Further study found that compared with $\mathrm{HP}$ - patients, $\mathrm{HP}+$ patients showed reduced sperm concentration, motility, and fertility index [9]. All the above studies indicate that HP infection may be a deteriorating factor for sperm quality, which deserves further investigation and treatment. However, most studies are from Italy, and additional data from different ethnicities may provide more robust evidence.

In females, there is a possible association between HP infection and polycystic ovarian syndrome (PCOS). A study from Turkey reported that the proportion of HP seropositivity was almost doubled in the PCOS population [10]. It is speculated that HP infection may lead to the release of certain substances or stimulate the immune response of the host, leading to the occurrence of PCOS. PCOS is manifested by increased ovarian reserve, while decreased ovarian reserve is an even worse problem that is difficult to treat. Since anti-Müllerian hormone (AMH) is an excellent indicator of ovarian reserve, we plan to investigate the association between HP infection and AMH.

Overall, there appears to be an association between HP infection and infertility, but available support is not sufficient and thus requires further validation. The purpose of the present study is as follows: (1) to investigate the correlation between HP infection and sperm quality in males and (2) to explore the association between HP infection and ovarian reserve in females.

\section{Methods}

\section{Population of study}

From January 2016 to June 2019, patients aged 20-50 years who came to the Second Hospital of
Zhejiang University School of Medicine due to abdominal discomfort and underwent HP testing were included in this study. Among them, 565 had plans for pregnancy and had performed sperm analysis or ovarian reserve tests in the past three months, who were involved for further analyses (Fig. 1).

\section{Detection of HP infection}

The ${ }^{13} \mathrm{C}$-urea breath test (UBT) or ${ }^{14} \mathrm{C}$-UBT was used to examine HP infection. Two breath samples were collected before and after ingestion of a ${ }^{13} \mathrm{C}$-urea (RichenForce, Beijing, China) or ${ }^{14} \mathrm{C}$-urea (Xinke, Shanghai, China) reagent dissolved in water. For the ${ }^{13} \mathrm{C}$-urea breath test, a change over baseline value greater than 4.0 delta over baseline (DOB) was taken as a positive result ( $\mathrm{HP}+$ ). For ${ }^{14} \mathrm{C}$-UBT, a result greater than $100 \mathrm{DPM}$ was taken as a positive result $(\mathrm{HP}+)$.

\section{Detection of sperm parameters}

Sperm samples were collected with sterile containers by masturbation after 2-7 days of sexual abstinence. After liquefaction at $37{ }^{\circ} \mathrm{C}$ for $30 \mathrm{~min}$, routine parameters including sperm concentration and motility were examined with a computer-assisted sperm analysis (CASA) system (WLJY-9000, Beijing, China) according to World Health Organization guidelines [11]. Sperm morphology was assessed by the Papanicolaou staining modified for spermatozoa according to World Health Organization guidelines [11].

\section{Detection of serum AMH and sex hormones}

Serum AMH was tested with the electrochemiluminescence method by an Elecsys ${ }^{\circledR}$ AMH from Roche Diagnostics on a Roche Cobas e602 analyzer. The total imprecision for the assays was $1.2 \%$ at a level of $1.19 \mathrm{ng} /$ $\mathrm{mL}$ with a measuring range of $0.01-23 \mathrm{ng} / \mathrm{mL}$. Serum

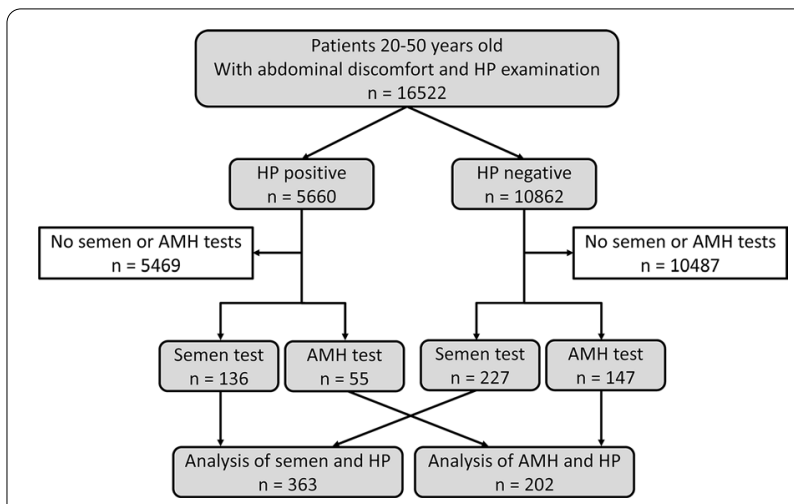

Fig. 1 Flow chart of the present study. HP: Helicobacter pylori; AMH: anti-Müllerian hormone 
sex hormone levels were detected with the electrochemiluminescence method by kits from Siemens Healthcare Diagnostics Inc.. The total imprecision for the assays was $3.0 \%$ at a level of $10,585 \mathrm{pmol} / \mathrm{L}$ for estradiol $\left(\mathrm{E}_{2}\right)$, $12.6 \%$ at a level of $0.37 \mathrm{nmol} / \mathrm{L}$ for testosterone (T), $2.7 \%$ at a level of $4.2 \mathrm{IU} / \mathrm{L}$ for luteinizing hormone (LH), 3.9\% at a level of $6.9 \mathrm{IU} / \mathrm{L}$ for follicle-stimulating hormone (FSH), $4.8 \%$ at a level of $69.9 \mathrm{mIU} / \mathrm{L}$ for prolactin (PRL), and $12.7 \%$ at a level of $3.8 \mathrm{nmol} / \mathrm{L}$ for progesterone $(\mathrm{P})$. The measuring ranges were $43.6-11,010 \mathrm{pmol} / \mathrm{L}$ for $\mathrm{E}_{2}$, 0.24-52.05 nmol/L for T, $0.07-200 \mathrm{IU} / \mathrm{L}$ for $\mathrm{LH}, 0.3-$ $200 \mathrm{IU} / \mathrm{L}$ for FSH, 6.4-4240 mIU/L for PRL, and 0.67$190.8 \mathrm{nmol} / \mathrm{L}$ for $\mathrm{P}$.

\section{Search strategy and data extraction}

To search for studies investigating the correlation between HP and sperm parameters, two reviewers independently searched the studies published in English via three databases, including PubMed, Embase, and Cochrane CENTRAL, until June 30, 2019. Articles were identified through computerized searches using the keywords as follows: ("semen analysis" OR "sperm count" OR "sperm motility") AND ("Helicobacter pylori" OR "Campylobacter pylori"). Meanwhile, we hand-searched the references listed in the achieved papers to obtain additional studies.

Two reviewers extracted the common characteristics and outcome parameters of the searched manuscripts independently. The common characteristics included the name of the first author, publication year, country, and number of patients. The clinical outcomes included sperm concentration and progressive motility percentage (PR).

\section{Statistical analysis}

Analyses were performed by using the SPSS 19.0 statistics package (SPSS, Chicago, IL, USA). Continuous variables are expressed as the mean values \pm standard deviation (SD). Student's t test was used for comparisons between two groups. Pearson correlation analysis was performed to analyze the relationship between two variables. A $P$ value of $<0.05$ was considered statistically significant.

Data from our hospital and previously published results were pooled and calculated together by Review Manager Software (RevMan Version 5.3). When the mean and SD were not provided in the published article, we used formulas to estimate them [12-14]. The results were presented as the mean difference (MD) and 95\% confidence interval (CI), and statistical significance was calculated by the $\mathrm{Z}$ test. If there was no serious heterogeneity ( $P$ value $\geq 0.1$ by the $Q$ test), a fixed-effects model (FEM) was applied for calculation, and if there was serious heterogeneity, a random-effects model (REM) was applied [15].

\section{Results}

Baseline characteristics of the involved population.

As shown in Fig. 1, a total of 16,522 patients who underwent the HP test were included in this study. Among these patients, $34.26 \%$ (5660) were HP positive. Among the patients with HP infection, 136 underwent the sperm test, and 55 underwent the AMH test. Among the patients without HP infection, 227 underwent the sperm test, and 147 underwent the AMH test. Finally, 363 were involved in the analysis between sperm and HP and 202 between AMH and HP.

As shown in Table 1, the baseline characteristics were similar in both the sperm and AMH analyses. In the analysis of sperm and HP, there was no significant difference in age, weight, height, or body mass index (BMI) between the $\mathrm{HP}+$ and $\mathrm{HP}-$ groups $(P>0.05)$. Similarly, in the analysis of AMH and HP, no significant difference was found in age, weight, height, or BMI between the HP+ and $\mathrm{HP}$ - groups $(P>0.05)$.

\section{Comparison of sperm parameters between groups with or without HP infection}

As shown in Table 1, the mean sperm concentration was $53.00 \times 10^{6} \mathrm{Sp} / \mathrm{mL}$ and $53.90 \times 10^{6} \mathrm{Sp} / \mathrm{mL}$ in the $\mathrm{HP}+$ and $\mathrm{HP}$ - groups, respectively, with no significant difference $(P>0.05)$. Sperm PR was also similar between the $\mathrm{HP}+$ and HP - groups (39.39\% vs. $39.92 \%)$, with no significant difference $(P>0.05)$. There was no difference in either normal sperm morphology percentage or sperm head defects $(P>0.05)$.

To further exclude the impact of age, we divided the population into subgroups of 20-24, 25-29, 30-34, $35-39,40-44$, and $45-50$ years of age. As shown in Fig. 2a, c, there was no significant difference in sperm concentration or PR between the HP+ and HP- groups for any age group $(P>0.05)$. As shown in Fig. $2 b, d$, in both the HP+ and HP- groups, there was no significant correlation between sperm concentration and age or between PR and age $(P>0.05)$.

\section{Comparison of AMH and sex hormones between groups with or without HP infection}

As shown in Table 1, the mean serum AMH level was 3.49 in the HP+ group and 3.25 in the HP- group, with no significant difference $(P>0.05)$. No difference was found between $\mathrm{HP}+$ and $\mathrm{HP}-$ groups in serum $\mathrm{E}_{2}, \mathrm{~T}, \mathrm{LH}$, FSH, PRL, or P levels $(P>0.05)$.

As shown in Fig. 2e, there was no significant difference in AMH level between the HP+ and $\mathrm{HP}$ - groups in every age span $(P>0.05)$. Meanwhile, AMH correlated 
Table 1 Characteristics of the present study

\begin{tabular}{|c|c|c|c|c|c|c|}
\hline \multirow[t]{2}{*}{ Characteristics } & \multicolumn{3}{|c|}{ Sperm and HP } & \multicolumn{3}{|l|}{$\mathrm{AMH}$ and $\mathrm{HP}$} \\
\hline & $\begin{array}{l}\mathrm{HP}+ \\
\mathrm{n}=136\end{array}$ & $\begin{array}{l}\mathrm{HP}- \\
n=227\end{array}$ & $P$ & $\begin{array}{l}\mathrm{HP}+ \\
n=55\end{array}$ & $\begin{array}{l}\mathrm{HP}- \\
n=147\end{array}$ & $P$ \\
\hline Age $(y)$ & $31.08 \pm 4.28$ & $31.19 \pm 4.52$ & 0.822 & $32.89 \pm 6.82$ & $34.27 \pm 6.83$ & 0.202 \\
\hline Weight (kg) & $69.72 \pm 10.05$ & $69.58 \pm 9.82$ & 0.898 & $52.91 \pm 8.57$ & $52.85 \pm 7.01$ & 0.961 \\
\hline Height (m) & $1.74 \pm 0.06$ & $1.74 \pm 0.06$ & 0.838 & $1.61 \pm 0.05$ & $1.61 \pm 0.04$ & 0.546 \\
\hline $\mathrm{BMI}\left(\mathrm{kg} / \mathrm{m}^{2}\right)$ & $22.98 \pm 2.78$ & $22.97 \pm 2.78$ & 0.967 & $20.26 \pm 2.72$ & $20.39 \pm 2.50$ & 0.757 \\
\hline Conc. $\left(\mathrm{Sp} / \mathrm{ml} \times 10^{6}\right)$ & $53.00 \pm 42.36$ & $53.90 \pm 46.95$ & 0.855 & NA & NA & NA \\
\hline PR (\%) & $39.39 \pm 18.61$ & $39.92 \pm 18.81$ & 0.793 & NA & NA & NA \\
\hline Normal (\%) & $6.73 \pm 3.97$ & $6.63 \pm 4.43$ & 0.865 & NA & NA & NA \\
\hline Head (\%) & $86.61 \pm 8.71$ & $84.77 \pm 13.44$ & 0.248 & NA & NA & NA \\
\hline AMH (ng/ml) & NA & NA & NA & $3.49 \pm 3.03$ & $3.25 \pm 2.82$ & 0.605 \\
\hline E2 (pmol/L) & NA & NA & NA & $194.46 \pm 78.53$ & $192.56 \pm 68.24$ & 0.906 \\
\hline $\mathrm{T}(\mathrm{nmol} / \mathrm{L})$ & NA & NA & NA & $0.94 \pm 0.71$ & $1.12 \pm 0.78$ & 0.118 \\
\hline LH (IU/L) & NA & NA & NA & $6.31 \pm 4.32$ & $4.98 \pm 3.32$ & 0.106 \\
\hline FSH (IU/L) & NA & NA & NA & $8.36 \pm 3.84$ & $8.38 \pm 3.43$ & 0.978 \\
\hline PRL (m/U/L) & NA & NA & NA & $256.19 \pm 154.03$ & $234.81 \pm 129.36$ & 0.489 \\
\hline $\mathrm{P}(\mathrm{nmol} / \mathrm{L})$ & NA & NA & NA & $1.99 \pm 1.03$ & $1.65 \pm 0.97$ & 0.128 \\
\hline
\end{tabular}

BMI: body mass index; AMH: anti-Müllerian hormone; NA: not available; Conc.: concentration; PR: progressive motility; Normal: normal sperm morphology percentage; Head: sperm head defects; DFI: sperm DNA fragmentation index; $E_{2}$ : estradiol; T: testosterone; LH: luteinizing hormone; FSH: follicle-stimulating hormone; PRL: prolactin; P: progesterone

significantly negatively with age (Fig. 2f, for $\mathrm{HP}-$, Pearson correlation coefficient $=-0.482, P=0.000$; for $\mathrm{HP}+$, Pearson correlation coefficient $=-0.431, P=0.001$ ).

\section{Pooled analysis of the association between sperm parameters and HP infection}

Since 2010, six studies investigated the correlation between HP infection and sperm parameters, as listed in Table 2. Most studies found that sperm motility was reduced significantly in CagA+ patients $[6,7,16,17]$. The latest study found that sperm concentration and PR were reduced in the $\mathrm{HP}+$ population, and in the $\mathrm{CagA}+$ population PR was reduced further than in the CagA- population [9].

Five studies including 703 participants were pooled to compare $\mathrm{HP}+$ and $\mathrm{HP}$ - groups, and five studies including 210 participants were pooled to compare CagA+and CagA- groups. As shown in Fig. 3a, c, FEM analysis showed that there was no significant difference in sperm concentration between the $\mathrm{HP}+$ and $\mathrm{HP}-$ groups or between the CagA+and CagA - groups $(P>0.05$ for both). In the sperm motility analysis between $\mathrm{HP}+$ and $\mathrm{HP}-$, since serious heterogeneity $(P<0.01)$ was found, a REM was applied and suggested no significant difference in PR (Fig. 3b, 95\% CI -11.44 to 1.87 , $P=0.16)$. FEM analysis was applied to compare sperm PR between CagA + and CagA - groups, which suggested that PR was $16.18 \%$ lower in the CagA+group than in the CagA - group (Fig. 3d, 95\% CI -18.86 to -13.50 , $P<0.01)$.

\section{Discussion}

In the present study, no difference was found in sperm concentration or sperm motility between $\mathrm{HP}+$ and $\mathrm{HP}$ - groups. Further subgroup analyses confirmed that there was no significant difference in sperm parameters between $\mathrm{HP}+$ and $\mathrm{HP}$ - groups. Furthermore, we pooled our data and those of previous studies and found no significant difference in sperm concentration or motility, indicating that in the Chinese population, HP infection does not disturb spermatogenesis.

The results of previous studies were not consistent. Some suggested that sperm concentration and motility were reduced in $\mathrm{HP}+$ patients [9] and that treating HP could improve the quality of sperm [18], while some suggested no significant difference in sperm parameters between $\mathrm{HP}+$ and $\mathrm{HP}$ - groups [6, 7, 16, 17]. The inconsistent results may be due to different test methods and ethnicities investigated.

This is the first study that used UBT to detect HP infection and to investigate its relationship with sperm quality. In previous studies, HP infection was detected with a serology test by enzyme-linked immunosorbent assay (ELISA) and confirmed with western blotting (WB) [6, $7,9,16-18$, whereas in the present study, ${ }^{13} \mathrm{C}$ - and ${ }^{14} \mathrm{C}$ UBT were used to detect HP infection. UBT is the best 

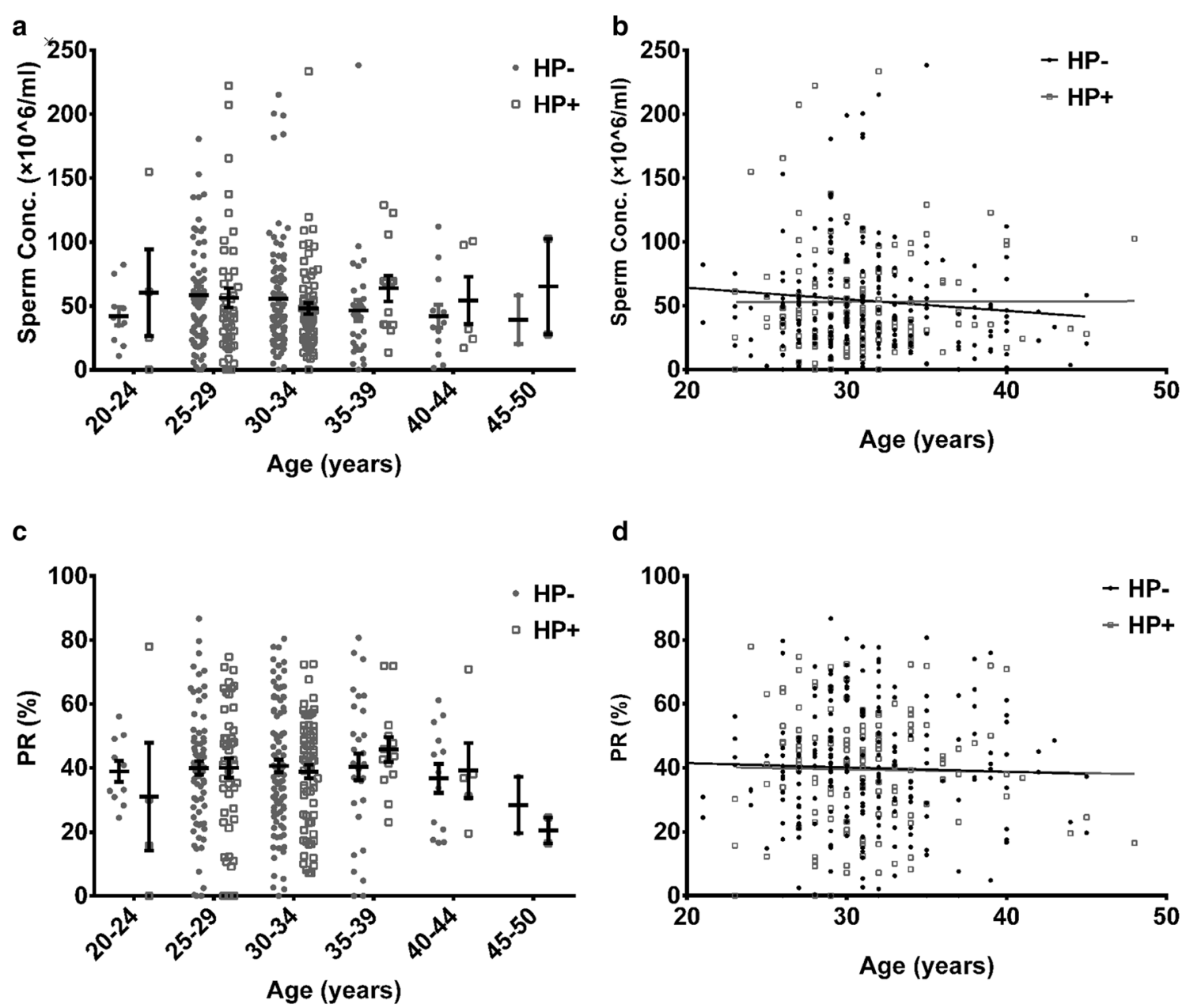

d

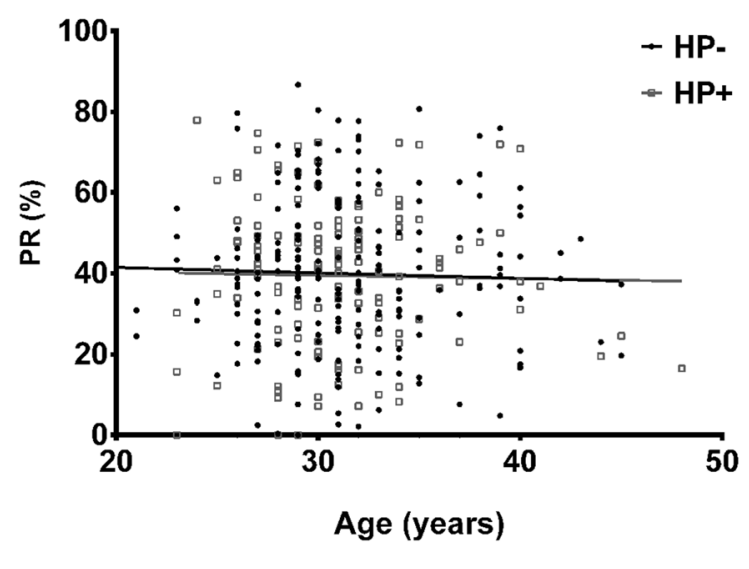

e

f
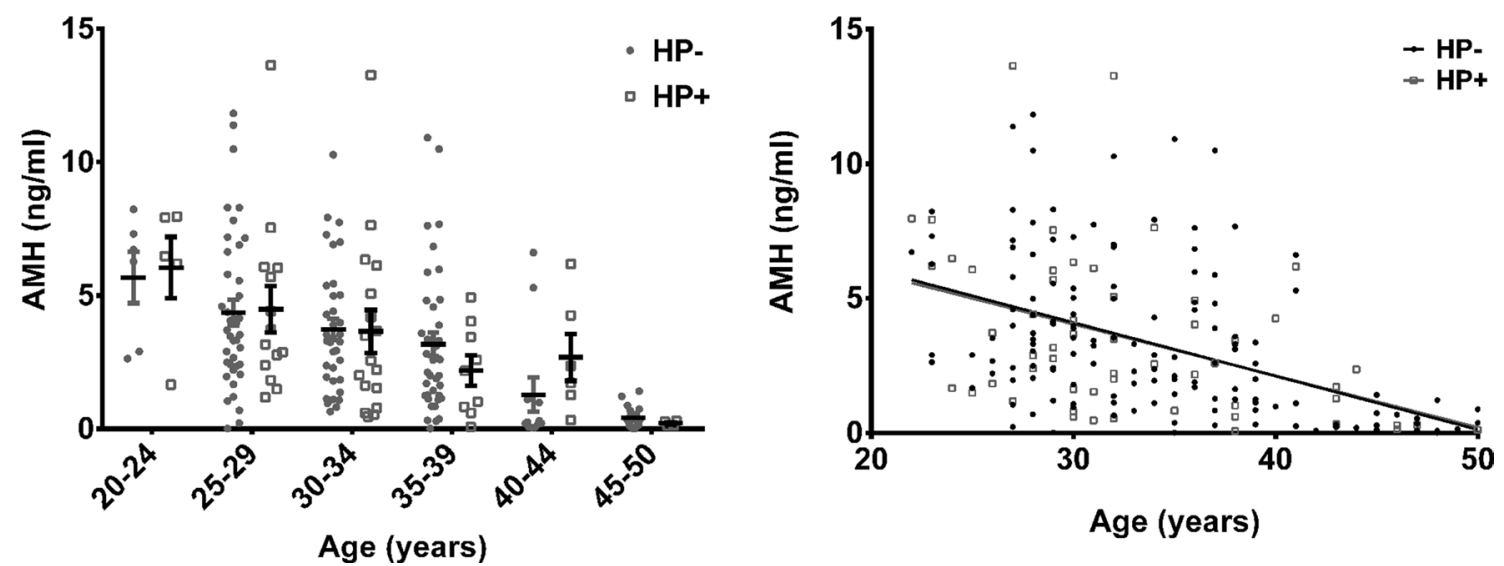

Fig. 2 Correlation between sperm parameters and anti-Müllerian hormone (AMH) and Helicobacter pylori (HP) infection. $\mathbf{a}$, b Correlation between AMH and HP. c, $\mathbf{d}$ Correlation between sperm concentration (Conc.) and HP. e, $\mathbf{f}$ Correlation between progressive motility percentage (PR) and HP 
Table 2 Previous studies investigating the impact of HP infection on sperm parameters

\begin{tabular}{|c|c|c|c|c|c|c|c|c|c|}
\hline \multirow[t]{2}{*}{ Study } & \multirow[t]{2}{*}{ Public year } & \multirow[t]{2}{*}{ Country } & \multicolumn{3}{|l|}{ HP (+ vs. - ) } & \multicolumn{3}{|c|}{ CagA (+ vs. - ) } & \multirow[t]{2}{*}{ Results } \\
\hline & & & Population & Conc & PR (\%) & Population & Conc & PR (\%) & \\
\hline Moretti [9] & 2017 & Italy & 32 vs. 41 & 38.0 vs. $55.0^{*}$ & 17.0 vs. $34.0^{* * *}$ & 20 vs. 12 & 33.4 vs. 42.5 & 10.5 vs. $22.5^{* * *}$ & $\begin{array}{l}\mathrm{HP}+\text { reduced conc. } \\
\text { and } \mathrm{PR}\end{array}$ \\
\hline Moretti [17] & 2015 & Italy & 28 vs.81 & 61 vs. 72 & 32 vs. 32 & 12 vs. 16 & 61 vs. 61.5 & 24 vs. $36.5^{*}$ & CagA+reduce $P R$ \\
\hline El-Garem [18] & 2014 & Egypt & NA & NA & NA & 22 vs. 201 & NA & NA & $\begin{array}{l}\text { HP treatment } \\
\text { improved PR }\end{array}$ \\
\hline Moretti [16] & 2013 & Italy & $\mathrm{NA}$ & $\mathrm{NA}$ & $\mathrm{NA}$ & 37 vs. 50 & 58 vs. 63 & 18 vs. $32^{* *}$ & Cag $A+$ reduced $P R$ \\
\hline Moretti [7] & 2012 & Italy & 27 vs. 51 & 94 vs. 72 & 32 vs. 30 & 11 vs. 16 & 65 vs. 94 & 24 vs. $38^{* *}$ & Cag $A+$ reduced $P R$ \\
\hline Collodel [6] & 2010 & Italy & 36 vs. 44 & 24.5 vs. 23.5 & 22 vs. 28.5 & 17 vs. 19 & 25.5 vs. 23 & 18 vs. $29^{*}$ & Cag $\mathrm{A}+$ reduced $\mathrm{PR}$ \\
\hline
\end{tabular}

NA: not available; Conc.: sperm concentration; PR: progressive motility; CagA: cytotoxin-associated gene A

${ }^{*} P<0.05 ;{ }^{* *} P<0.01 ;{ }^{* *} P<0.001$

noninvasive method for patients without gastric resection or proton pump inhibitor (PPI) treatment, with both high positive predictive value and negative predictive value [19-21]. A meta-analysis suggested that UBT had high diagnostic accuracy for detecting HP infection in patients with dyspepsia, with a pooled sensitivity of UBT in adult patients of $96 \%$ and a pooled specificity of $93 \%$ $[22,23]$. Another meta-analysis involving 34 studies with serology evaluation and 57 studies with UBT detection reported that the sensitivity of HP diagnosis was 0.94 for ${ }^{13} \mathrm{C}$-UBT, 0.92 for ${ }^{14} \mathrm{C}$-UBT, and 0.84 for serology tests. UBT showed a higher diagnostic accuracy than the serology test for HP infection diagnosis [24, 25]. Therefore, in this study, UBT was used, as it provides a more accurate HP diagnosis than serology tests.

Moreover, serology tests cannot distinguish between active and inactive infections [26]. In a letter from Caviglia et al., the authors emphasized that the presence of serological HP antibodies could only indicate previous exposure, not necessarily a current infection, and based on this, they recommended UBT as a direct diagnostic test [27]. Similarly, in the present study, UBT examination represented the status of current HP infection better than serology tests.

CagA is the major virulence factor in HP, encoding the CagA protein in the cag pathogenicity island [28]. HP infection can be divided into two isolates: CagAproducing strains $(\mathrm{CagA}+)$ and $\mathrm{CagA-nonproducing}$ strains (CagA-). Our meta-analysis of sperm motility and CagA-producing/nonproducing strain infection suggested that PR was $16.18 \%$ lower in the CagA+group than in the CagA- group. The underlying mechanism may be that CagA + HP infection induces overexpression of miR-543 and downregulation of the p14ARF tumor suppressor to inhibit autophagy and increase cytokine production, which induces inflammatory responses of HP accordingly [29-31]. Anti-CagA antibodies may block spermatozoa acrosomes and disturb fertilization [8].

The prevalence of the CagA genotype in HP infection varies significantly among different regions. In Western countries, CagA+strains comprise $50-60 \%$ of the $\mathrm{HP}+$ population, and in the Chinese population, CagA+ strains occupy nearly $100 \%$ of the HP+ population [32, 33]. Studies investigating the CagA status of Chinese HP strains with polymerase chain reaction (PCR) detected CagA genotypes in nearly all strains [34, 35]. Considering the high CagA positivity in the Chinese $\mathrm{HP}+$ population, the sperm concentration and motility should be weakened in HP+ patients, but the present study showed stable parameters. Further study investigating CagA antibody status should be performed to clarify the role of CagA in sperm quality.

The variability of sperm parameters after HP treatment is an interesting question. It was reported that after the treatment of HP, seminal HP IgA level decreased significantly, and meanwhile progressive sperm motility, nonprogressive sperm motility, and sperm normal forms increased significantly $(P=0.001)$ [18]. In the present study, sperm analyses were performed before HP test, and most patients with $\mathrm{HP}+$ suspended their plans of pregnancy after HP treatment. Therefore, we did not follow the sperm parameters.

In the present study, there was no difference in serum $\mathrm{AMH}$ level between $\mathrm{HP}+$ and HP- groups, which was confirmed with further age-divided subgroup analyses. Published results of the relationship between PCOS and HP infection are inconsistent. Yavasoglu et al. found that HP antibody positivity was significantly more common in the PCOS group than in the age-matched control group [10]. The possible explanation may be that the antigenic mimicry to HP antigens leads to an immune cross-reaction between HP antigens and the ovaries, inducing the onset of PCOS [36]. Nevertheless, 


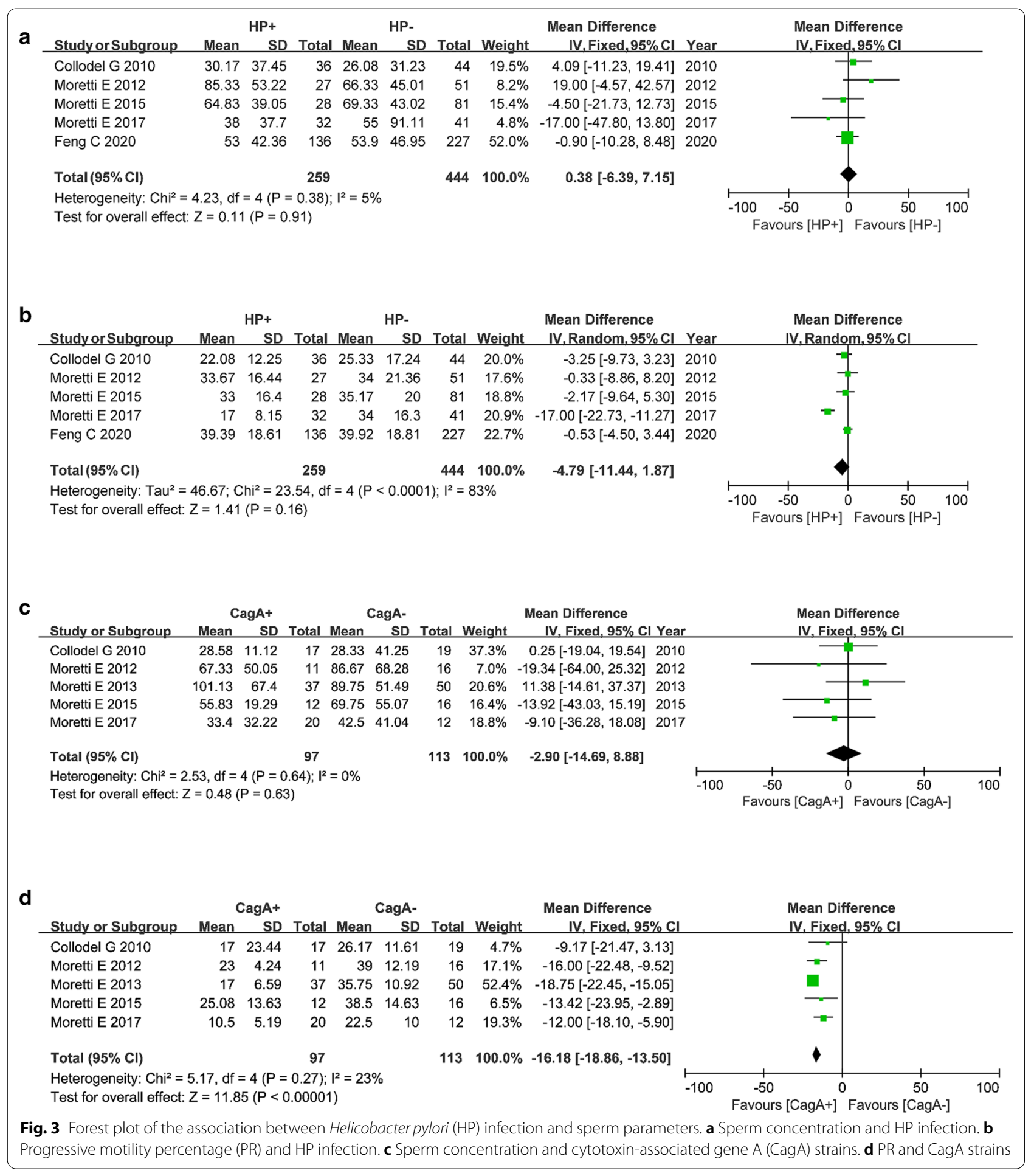

Tokmak et al. found no significant difference in HP IgG positivity between PCOS and non-PCOS groups [37]. AMH is a potential future substitute for detecting polycystic ovarian morphology (PCOM) and a useful biomarker for predicting the risk of PCOS [3840]. Our data indicated no correlation between PCOS and HP infection. Meanwhile, AMH is considered the best serum biomarker of ovarian reserve, reflecting the number of primordial follicles and its response to 
exogenous gonadotropins [41]. The present study indicates that ovarian reserve is stable with HP infection.
Received: 21 December 2019 Accepted: 24 September 2020

Published online: 26 November 2020

\section{Conclusion}

This is the first observation investigating the impact of $\mathrm{HP}$ infection on ovarian reserve, which found that HP infection was not related to the serum ovarian reserve biomarker AMH. In general, HP infection is not a crucial factor affecting sperm parameters or ovarian reserve.

\begin{abstract}
Abbreviations
DOB: Delta over baseline; $E_{2}$ : Estradiol; EPL: Early pregnancy loss; FEM: Fixedeffects model; FSH: Follicle-stimulating hormone; HP: Helicobacter pylori; LH: Luteinizing hormone; MD: Mean difference; P: Progesterone; PCOM: Polycystic ovarian morphology; PCOS: Polycystic ovarian syndrome; PCR: Polymerase chain reaction; PPI: Proton pump inhibitor; PR: Progressive motility percentage; PRL: Prolactin; REM: Random-effects model; SD: Standard deviation; T: Testosterone; UBT: Urea breath test; WB: Western blotting.
\end{abstract}

\section{Acknowledgements}

We are especially grateful to Ya-Wen Xing for his kind help in data collection.

\section{Authors' contributions}

CF, MJ, and JMS conceived and designed the study; CF, MJ and CCH enrolled the patients and collected the data; CF, PPL, SQY, and QPY analyzed the data; PPL and MJ reviewed the references and extracted the data; CF, MJ, and JMS organized and wrote the manuscript. All authors read and approved the final manuscript.

\section{Funding}

This work was financially supported by the following funding sources: the, including National Key R\&D Program of China (2018YFC1004900 to CF), National Natural Science Foundation of China (81871176 to CF, 81701461 to PPL, and 81671487 to MJ), and the Medical and Health Science and Technology Plan of Zhejiang Province (2018251579 to PPL) for the English language editing service costs, and article-processing charge. No funders had any role in the study design, data analysis of data, or writing manuscript.

\section{Availability of data and materials}

The data analyzed in this study are available from the corresponding author upon request.

\section{Ethics approval and consent to participate}

The present study was approved by the Ethics Committee of the Second Affiliated Hospital of Zhejiang University School of Medicine (IR2019001059). Since this was a retrospective observational study and no intervention was needed, no formal ethical approval or written consent was required, which was approved by the Ethics Committee of the Second Affiliated Hospital of Zhejiang University School of Medicine.

\section{Consent for publication}

Not applicable.

\section{Competing interests}

The authors declare that they have no competing interests.

\section{Author details}

${ }^{1}$ Department of Reproductive Medicine, The Second Affiliated Hospital of Zhejiang University School of Medicine, 88 Jiefang Road, Hangzhou 310009, Zhejiang, China. ${ }^{2}$ The Women's Hospital of Zhejiang University School of Medicine, Hangzhou 310006, Zhejiang, China. ${ }^{3}$ Department of Orthopedics, The First Affiliated Hospital of Zhejiang Chinese Medicine University, 54 Youdian Road, Hangzhou 310006, Zhejiang, China.

\section{References}

1. Franceschi F, Covino M, Roubaud BC. Review: Helicobacter pylori and extragastric diseases. Helicobacter. 2019;24(Suppl 1):e12636.

2. Ražuka-Ebela D, Giupponi B, Franceschi F. Helicobacter pylori and extragastric diseases. Helicobacter. 2018;23(Suppl 1):e12520.

3. Figura N, Piomboni P, Ponzetto A, Gambera L, Lenzi C, Vaira D, et al. Helicobacter pylori infection and infertility. Eur J Gastroenterol Hepatol. 2002;14(6):663-9.

4. Kurotsuchi S, Ando H, Iwase A, Ishida Y, Hamajima N, Kikkawa F. The plausibility of Helicobacter pylori-related infertility in Japan. Fertil Steril. 2008;90(3):866-8.

5. Hajishafiha M, Ghasemi-Rad M, Memari A, Naji S, Mladkova N, Saeedi V. Effect of Helicobacter pylori infection on pregnancy rates and early pregnancy loss after intracytoplasmic sperm injection. Int J Womens Health. 2011;3:329-35.

6. Collodel G, Moretti E, Campagna MS, Capitani S, Lenzi C, Figura N. Infection by CagA-positive Helicobacter pylori strains may contribute to alter the sperm quality of men with fertility disorders and increase the systemic levels of TNF-alpha. Dig Dis Sci. 2010;55(1):94-100.

7. Moretti E, Collodel G, Campagna MS, Franci MB, lacoponi F, Mazzi L, et al. Influence of Helicobacter pylori infection on levels of ghrelin and obestatin in human semen. J Androl. 2012;33(5):938-43.

8. Ponzetto A, Holton J. Extragastric diseases correlated with Helicobacter pylori. Helicobacter. 2019;24(1):e12549.

9. Moretti E, Figura N, Campagna MS, lacoponi F, Gonnelli S, Collodel G. Infectious burden and semen parameters. Urology. 2017;100:90-6.

10. Yavasoglu I, Kucuk M, Cildag B, Arslan E, Gok M, Kafkas S. A novel association between polycystic ovary syndrome and Helicobacter pylori. Am J Med Sci. 2009;338(3):174-7.

11. WHO laboratory manual for the examination and processing of human semen (Fifth edition). https://www.who.int/reproductivehealth/publi cations/infertility/9789241547789/en/. 2010.

12. Wan $X$, Wang W, Liu J, Tong T. Estimating the sample mean and standard deviation from the sample size, median, range and/or interquartile range. BMC Med Res Methodol. 2014;14:135.

13. Luo D, Wan X, Liu J, Tong T. Optimally estimating the sample mean from the sample size, median, mid-range, and/or mid-quartile range. Stat Methods Med Res. 2018;27(6):1785-805.

14. Higgins JPT GS. Cochrane handbook for systematic reviews of interventions version 5.1.0. The Cochrane Collaboration, 2011.

15. Lau J, loannidis JP, Schmid CH. Quantitative synthesis in systematic reviews. Ann Intern Med. 1997;127(9):820-6.

16. Moretti E, Collodel G, Mazzi L, Campagna MS, Figura N. CagA-positive Helicobacter pylori infection and reduced sperm motility, vitality, and normal morphology. Dis Mark. 2013;35(4):229-34.

17. Moretti E, Figura N, Campagna MS, Gonnelli S, lacoponi F, Collodel G. Sperm parameters and semen levels of inflammatory cytokines in Helicobacter pylori-infected men. Urology. 2015;86(1):41-6.

18. El-Garem Y, El-Sawy M, Mostafa T. Seminal Helicobacter pylori treatment improves sperm motility in infertile asthenozoospermic men. Urology. 2014;84(6):1347-50.

19. Tonkic A, Tonkic M, Lehours P, Mégraud F. Epidemiology and diagnosis of Helicobacter pylori infection. Helicobacter. 2012;17(Suppl 1):1-8.

20. Kawai S, Arai K, Lin Y, Nishiyama T, Sasakabe T, Wang C, et al. Comparison of the detection of Helicobacter pylori infection by commercially available serological testing kits and the ${ }^{13} \mathrm{C}$-urea breath test. J Infect Chemother. 2019;25(10):769-73.

21. Kusano C, Gotoda T, Ikehara H, Suzuki S, Shibuya H, Horii T, et al. The accuracy of the serum antibody test for Helicobacter pylori infection among junior high school students. Digestion. 2019. https://doi. org/10.1159/00050290.

22. Mentis A, Lehours P, Mégraud F. Epidemiology and Diagnosis of Helicobacter pylori infection. Helicobacter. 2015;20(Suppl 1):1-7. 
23. Ferwana M, Abdulmajeed I, Alhajiahmed A, Madani W, Firwana B, Hasan $R$, et al. Accuracy of urea breath test in Helicobacter pylori infection: metaanalysis. World J Gastroenterol. 2015;21(4):1305-14.

24. Best LM, Takwoingi Y, Siddique S, Selladurai A, Gandhi A, Low B, et al. Non-invasive diagnostic tests for Helicobacter pylori infection. Cochrane Database Syst Rev. 2018;3:CD012080.

25. Makristathis A, Hirschl AM, Mégraud F, Bessède E. Review: Diagnosis of Helicobacter pylori infection. Helicobacter. 2019;24(Suppl 1):e12641.

26. Miftahussurur M. Noninvasive Helicobacter pylori diagnostic methods in Indonesia. Gut Liver. 2019. https://doi.org/10.5009/gn/19264.

27. Caviglia GP, Fagoonee S, Pellicano R. Re: El-Garem et al.: Seminal helicobacter pylori treatment improves sperm motility in infertile asthenozoo spermic men (Urology 2014;84:1347-1350). Urology. 2015;85(5):1217.

28. Park JY, Forman D, Waskito LA, Yamaoka Y, Crabtree JE. Epidemiology of Helicobacter pylori and CagA-positive infections and global variations in gastric cancer. Toxins (Basel). 2018;10(4):E163.

29. Li N, Tang B, Jia YP, Zhu P, Zhuang Y, Fang Y, et al. Helicobacter pylori CagA protein negatively regulates autophagy and promotes inflammatory response via c-Met-PI3K Akt-mTOR signaling pathway. Front Cell Infect Microbiol. 2017:7:417.

30. Shi Y, Yang Z, Zhang T, Shen L, Li Y, Ding S. SIRT1-targeted miR-543 autophagy inhibition and epithelial-mesenchymal transition promotion in Helicobacter pylori CagA-associated gastric cancer. Cell Death Dis. 2019;10(9):625.

31. Horvat A, Noto JM, Ramatchandirin B, Zaika E, Palrasu M, Wei J, et al. Helicobacter pylori pathogen regulates p14ARF tumor suppressor and autophagy in gastric epithelial cells. Oncogene. 2018;37(37):5054-65.

32. Yamaoka Y. Mechanisms of disease: Helicobacter pylori virulence factors. Nat Rev Gastroenterol Hepatol. 2010;7(11):629-41.

33. Vilaichone RK, Mahachai V, Tumwasorn S, Wu JY, Graham DY, Yamaoka Y. Molecular epidemiology and outcome of Helicobacter pylori infection in Thailand: a cultural cross roads. Helicobacter. 2004;9(5):453-9.
34. Zhou J, Zhang J, Xu C, He L. cagA genotype and variants in Chinese Helicobacter pylori strains and relationship to gastroduodenal diseases. $J$ Med Microbiol. 2004;53(Pt 3):231-5.

35. Yin L, Liu F, Guo C, Wang Q, Pan K, Xu L, et al. Analysis of virulence diversity of 73 Helicobacter pylori strains isolated in Guizhou province. China Mol Med Rep. 2018;18(5):4611-20

36. Yavasoglu I, Kucuk M. Anti-Helicobacter pylori antibodies and polycystic ovary syndrome. Eur J Obstet Gynecol Reprod Biol. 2012;163(2):243.

37. Tokmak A, Doğan Z, Sarıkaya E, Timur H, Kekilli M. Helicobacter pylori infection and polycystic ovary syndrome in adolescent and young adult patients. J Obstet Gynaecol Res. 2016;42(12):1768-72.

38. Abbara A, Eng PC, Phylactou M, Clarke SA, Hunjan T, Roberts R, et al. Anti-Müllerian hormone (AMH) in the diagnosis of menstrual disturbance due to polycystic ovarian syndrome. Front Endocrinol (Lausanne). 2019;10:656.

39. Calzada M, López N, Noguera JA, Mendiola J, Hernández Al, Corbalán $\mathrm{S}$, et al. AMH in combination with SHBG for the diagnosis of polycystic ovary syndrome. J Obstet Gynaecol. 2019;39(8):1130-6.

40. Teede H, Misso M, Tassone EC, Dewailly D, Ng EH, Azziz R, et al. AntiMüllerian hormone in PCOS: a review informing international guidelines. Trends Endocrinol Metab. 2019;30(7):467-78.

41. Hansen KR, Hodnett GM, Knowlton N, Craig LB. Correlation of ovarian reserve tests with histologically determined primordial follicle number. Fertil Steril. 2011;95(1):170-5.

\section{Publisher's Note}

Springer Nature remains neutral with regard to jurisdictional claims in published maps and institutional affiliations.
Ready to submit your research? Choose BMC and benefit from:

- fast, convenient online submission

- thorough peer review by experienced researchers in your field

- rapid publication on acceptance

- support for research data, including large and complex data types

- gold Open Access which fosters wider collaboration and increased citations

- maximum visibility for your research: over $100 \mathrm{M}$ website views per year

At BMC, research is always in progress.

Learn more biomedcentral.com/submissions 